\title{
Do mindful people set better goals? Investigating the relation between trait mindfulness, self-concordance, and goal progress
}

\author{
Aidan P. J. Smyth \\ Carleton University \\ Marina Milyavskaya \\ Carleton University
}

\author{
Kaitlyn M. Werner \\ University of Toronto \\ Anne C. Holding, Richard Koestner \\ McGill University
}

\begin{abstract}
The present research examined the relation between trait mindfulness, self-concordance, and goal progress. We hypothesized that mindfulness would be positively associated with setting self-concordant goals (Studies 1-3), which would in turn predict greater goal progress (Studies 2 and 3). An internal mini meta-analysis $(N=1522)$ indicates that mindfulness had a small, positive association with self-concordant goal setting, $r=.14, p<.001$. Two longitudinal studies (Studies 2 and 3) found a small indirect effect of mindfulness on goal progress through self-concordance, although this was marginal in Study 2. In addition, Studies 2 and 3 found that mindfulness predicted increases in goal self-concordance over time. These findings provide support for the proposition that mindful people set "better" goals.
\end{abstract}

Data, analysis code, supplementary material: osf.io/p97jh

Keywords: mindfulness, self-concordance, goal setting, goal pursuit, goal progress, goal internalization

\section{In Press at Journal of Research in Personality}

\begin{abstract}
Please cite this article as: Smyth, A. P. J., Werner, K. M., Milyavskaya, M., Holding, A. C., \& Koestner, R. (in-press). Do mindful people set better goals? Investigating the relation between trait mindfulness, self-concordance, and goal progress. Journal of Research in Personality,
\end{abstract}

One of life's universal quests involves discovering what is really important to us and what we truly care about. Indeed, uncovering one's "purpose" in life is a perennial theme in film and literature and a common intervention in the self-help genre. In recent decades, research on personal goal pursuit has begun to address this essential topic. The self-concordance model (Sheldon \& Elliot, 1999) focuses on the importance of pursuing goals that truly reflect an individual's authentic self. The purpose of the present research was to investigate whether trait mindfulness is positively associated with self-concordant goal setting, and in turn greater goal progress.

Self-concordance has been conceptualized as the extent to which individuals' goals reflect their personal values and interests as opposed to internal or external pressures (Sheldon \& Elliot, 1999). Self-concordance also involves the match between individuals' implicit personalities and their explicit goals (i.e., the congruence between implicit and explicit motives;
Sheldon, Prentice, Halusic, \& Schüler, 2015). Drawing from self-determination theory (Deci \& Ryan, 2000), goal self-concordance has been operationalized as the extent to which goals are pursued for autonomous relative to controlled reasons (Sheldon \& Elliot, 1999). Autonomous reasons for pursuing a goal include doing so because it is interesting or enjoyable (intrinsic), because the goal is inherently important to the individual (identified), and because the goal represents one's values (integrated). On the other hand, controlled reasons include pursuing a goal to avoid feelings of shame or guilt (introjection) and because one will get a concrete reward, such as money (external).

When people pursue goals that are aligned with their underlying values, talents, interests, and needs (i.e., self-concordant goals), they are more likely to attain their goals (Koestner, Lekes, Powers, \& Chicoine, 2002; Koestner, Otis, Powers, Pelletier, \& Gagnon, 2008; Sheldon \& Elliot, 1999). Moreover, pursuing and attaining self-concordant goals affords experiences of 
autonomy, competence, and relatedness, which are essential to well-being (Sheldon \& Elliot, 1999; Deci $\&$ Ryan, 2000). Conversely, pursuing non-concordant goals can lead people to waste time and energy on goals that, even if attained, will not benefit their well-being or development (Sheldon \& Elliot, 1999; cf. Werner \& Milyavskaya, 2018). In fact, pursuing non-concordant goals can result in action crises and symptoms of depression (Holding, Hope, Harvey, Marion Jetten, \& Koestner, 2017). Thus, self-concordant goal setting is an important self-perceptual skill with significant consequences for mental health, well-being, and maturation (Sheldon, 2014).

Accumulating evidence supports the benefits of pursuing and attaining self-concordant goals (see Sheldon, 2014); however, less research has investigated intraindividual factors that may promote self-concordant goal selection (Donald et al., 2019). Sheldon (2014) posited that self-concordance is enhanced by intrapersonal variables that increase accurate self-insight. Consistent with this idea, Burton (2008) demonstrated that participants instructed to "follow their heart" and "listen to their gut" (i.e., attend to their internal compass and use faith in intuition) while setting goals, set goals that were more self-concordant compared to participants instructed to "be very rational." These findings may reflect the importance of paying attention to holistic information including subtle, somatic cues that provide important insights for identifying self-appropriate goals. Similarly, mindfulness has been theorized to enhance self-concordant goal selection (Sheldon, 2014).

Mindfulness involves paying attention to the present moment, on purpose, in an open and nonjudgmental manner (Brown \& Ryan, 2003; Kabat-Zinn, 2003). Thus, mindfulness promotes awareness of both our internal and external worlds. Awareness of our internal world, or self-awareness, consists of our physical sensations as well as our psychological experience, such as our thoughts, feelings, emotions, and desires. The enhanced self-awareness that is associated with greater dispositional mindfulness (Brown \& Ryan, 2003) may be particularly relevant to the selection of self-concordant goals because people must attend to their inner experience in order to select and pursue reflectively considered goals (Brown, Ryan, \& Creswell, 2007; Sheldon \& Kasser, 2001).

Given that mindful individuals are more attentive to their thoughts and emotions, they may have greater insight into their core values and interests (Shapiro, Carlson, Astin, \& Freedman, 2006), which should facilitate the selection of self-concordant goals
(Sheldon, 2014). Conversely, automatic processing, that is, acting without attention or awareness, often limits consideration of behaviours or goals that are in line with our needs, values, and interests (Brown \& Ryan, 2003; Ryan, Kuhl, \& Deci, 1997). Moreover, in attending to internal, somatic cues, mindful individuals may be better able to recognize when they are engaged in goals and tasks that are self-concordant, and when they are not. For example, in an experience sampling study, Brown and Ryan (2003) reported that trait mindfulness positively predicted autonomously motivated action in everyday life (i.e., engaging in activities because they are interesting, meaningful and/or in line with one's values). They also found that when participants reported experiencing state mindfulness, they were more likely to be acting autonomously.

A growing body of research generated in the context of self-determination theory supports a link between mindfulness and more adaptive motivational qualities (Donald et al., 2019). For example, a recent metaanalysis that included both correlational $(n=83)$ and intervention $(n=21)$ studies, reports that mindfulness is positively associated with autonomous forms of motivation and negatively associated with controlled forms of motivation and amotivation (Donald et al., 2019). These associations span a variety of domains including individuals' motivational orientation towards everyday activities, specific health behaviours, and various work and laboratory tasks. Moreover, intervention studies provide insight into the direction of the relationship between mindfulness and autonomous motivation, in particular, and suggest that mindfulness promotes a more autonomous orientation (Donald et al., 2019). Nonetheless, limited research has investigated mindfulness in the context of personal goal pursuit. The majority of the research on mindfulness and motivation has focused on individuals'

motivation towards goals assigned by the researchers (Ryan \& Deci, 2017) and thus provides little insight into the goals that mindful individuals are likely to set and pursue on their own. Given that mindfulness is associated with enhanced awareness and more adaptive motivational qualities, mindful individuals may be better able to recognize and select goals that are congruent with their authentic selves.

Previous research appears to provide preliminary evidence for this notion (Grégoire, Bouffard, \& Vezeau, 2012). Grégoire et al. (2012) found that individuals who reported higher trait mindfulness were more likely to be pursuing goals that were 
"self-determined", 1 which in turn predicted greater psychological and subjective well-being. While these findings are encouraging, the authors acknowledged some important limitations to their research. First, their investigation relied on a small, homogenous sample (114 French-speaking undergraduate university students; $90 \%$ female); thus, the findings may not generalize to other populations. Second, the researchers used a cross-sectional design to examine the mediating role of self-determined goals in the relationship between mindfulness and well-being; therefore, these relationships were not investigated over time.

Another important limitation is that the researchers had participants report on goals that they were already engaged in, rather than newly set goals. It is possible that participants' ratings of goal self-determination changed over the course of their goal pursuit (i.e., from the time they first set their goals until the time they reported on them in the study; Holding, Hope, Verner-Fillion, \& Koestner, 2019; Moore et al., 2018). Accordingly, it is also possible that the relationships between their variables of interest - namely trait mindfulness and goal self-determination, could change over time. It is worth noting that this limitation applies to the literature on mindfulness and motivation more generally, which has frequently focused on individuals' motivation towards goals and behaviours that they were already engaged in or that they typically engage in (e.g., Christie, Atkins, \& Donald et al., 2017; Warren \& Wray-Lake, 2017).

The idea that ratings of goal self-concordance (or goal self-determination) could change over the course of an individual's goal pursuit is reflected in the concepts of internalization and integration described in self-determination theory. According to selfdetermination theory, people engage in behaviours for a variety of different reasons, many of which are extrinsic in nature (Ryan \& Deci, 2017). Internalization can be understood as "the process of taking in values, beliefs, or behavioural regulations from external sources and transforming them into one's own" (Ryan \& Deci, 2017, p. 180). For example, a student who enters university in a science program at the insistence of her parents may over time come to enjoy and see the value of pursuing this course of study. The concepts of internalization and self-concordance are similar, both conceptually and operationally, in that they both reflect measures relative autonomy. However, the process of internalization is inherently longitudinal and therefore differs from goal self-concordance, which can be measured at one point in time (e.g., when individuals set their goals). Internalization is especially likely to occur when individuals are situated in need-supporting environments (Ryan \& Deci, 2017) or when individuals become more cognizant of the value of their goals. Given that mindfulness is thought to facilitate the process of integrating external regulations that are in line with one's values (Ryan \& Deci, 2017), it is possible that mindfulness facilitates the internalization of individuals' own personal goals over time. This proposition has yet to be empirically tested.

Despite the wide-ranging benefits associated with mindfulness, to date, no research has investigated the relationship between trait mindfulness and personal goal progress. Since mindfulness is theorized to predict goal self-concordance, it is also possible that mindfulness indirectly promotes goal progress. Previous research has shown that pursuing self-concordant goals can facilitate goal progress through increased effort (Koestner et al., 2002; Koestner et al., 2008; Sheldon \& Elliot, 1999), subjective ease (Werner, Milyavskaya, Foxen-Craft, \& Koestner, 2016), fewer obstacles, and decreased attraction to goal-disruptive temptations (Milyavskaya, Inzlicht, Hope, \& Koestner, 2015). If mindful individuals are more likely to set self-concordant goals, they may in turn be more likely to make progress on, and attain, their goals.

Mindfulness is also associated with a number of positive outcomes that may facilitate goal pursuit, such as enhanced self-regulation (Brown \& Ryan, 2003; Friese \& Hofmann, 2016), improved focus(Hafenbrack $\&$ Vohs, 2018), and more adaptive coping strategies for managing stress (Weinstein, Brown, \& Ryan, 2009). Some research, however, suggests that mindfulness may not be particularly helpful in the context of goal pursuit, and may in fact undermine individuals' amount of motivation towards goals (Hafenbrack \& Vohs, 2018). Nonetheless, this finding contrasts with substantial evidence linking mindfulness to more adaptive motivational qualities (Donald et al., 2019). It is thus important to investigate the relation between mindfulness, motivation, and personal goal progress.

continuum) and then subtracting the external and introjected scores from the intrinsic and identified scores, for each goal. On the other hand, goal selfconcordance is typically calculated by subtracting the average of the controlled items from the average of the autonomous items, for each goal. 


\section{Present Research}

Given that mindfulness is an intrapersonal variable that is associated with accurate self-insight and enhanced self-regulation, the purpose of the present research was to examine whether mindfulness can facilitate self-concordant goal setting and goal pursuit. Although mindfulness can be characterized as both a trait and a state, the present research focused on trait mindfulness since the cultivation of self-awareness required to select self-concordant goals is likely a longterm process that involves continued practice. $2 \mathrm{We}$ hypothesized that greater dispositional mindfulness would be positively associated with self-concordant goal setting (H1; Studies 1-3), and that mindfulness would have an indirect effect on goal progress through self-concordant goal setting (H2; Studies 2 and 3); although no hypothesis was made with regards to the correlation between mindfulness and goal progress, it is worth noting that a total effect is not necessary to establish mediation (Zhao, Lynch, \& Chen, 2010). Additional exploratory analyses were conducted to investigate whether mindfulness predicted increases in goal self-concordance over time (Studies 2 and 3).

\section{Statement of Transparency}

The data used in the present studies were all initially collected as part of larger studies on goal pursuit (i.e., this entire manuscript is comprised of secondary data analysis). Although we did not formally pre-register any of these studies prior to data collection, Study 3 served as a replication of Study 2 and so we posted a time-stamped version of our research questions, hypotheses, and analytical plan. Study materials, planned analyses, and output are available on the Open Science Framework (OSF). 3 Project link: osf.io/p97jh

\footnotetext{
2 We also conducted an experimental study where we attempted to manipulate state mindfulness. However, this study had some serious flaws, so that no conclusions could be drawn. We present the details of that study in an online supplement on OSF.

3 Data from the present research has not been posted publicly because it consists of large longitudinal datasets that were collected before we obtained participants' permission to publicly post anatomized data as part of our consent procedures.

4 Study 1 was conducted with two separate samples that were part of the same research project (Werner, 2019). One sample (Study 1a) was examined in the lab whereas the other sample (Study 1b) was examined online. Initially these samples were analysed separately. Study 1a showed
}

\section{Study 1}

As a first step, Study 1 used a cross-sectional design to examine whether trait mindfulness was positively associated with setting self-concordant goals.

\section{Method}

\section{Participants and Procedure}

Data from a previously collected study was used to test our first hypothesis. Participants in Study 1 were 799 undergraduate students $(69 \%$ women; $58 \%$ Caucasian; $M_{\text {age }}=20.54$ years, $S D=4.97$ ), all of whom took part in a larger study on goal pursuit.4 Participants $(n=25)$ were excluded if they did not complete the items for our variables of interest. Participants set three personal goals that they were going to pursue over the next week, rated their motivation for each goal, and completed a measure of trait mindfulness.

\section{Measures}

Goal Setting Task. The instructions for setting the personal goals were as follows:

"Personal goals are projects and concerns that people think about, plan for, carry out, and sometimes (though not always) complete or succeed at. They may be more or less difficult to implement; require only a few or a complex series of steps; represent different areas of a person's life; and be more or less time consuming, attractive, and urgent. Please list three personal goals that you plan to pursue over the upcoming week."

These instructions have been used in previous research on goal pursuit (Koestner et al., 2002; Werner et al., 2016). Some examples of goals that participants set include: "study for midterm", "workout", and "catch up on my readings".

\footnotetext{
a significant relationship whereas Study $1 \mathrm{~b}$ did not. We decided to combine these samples for the sake of readability. The output and write-up for Studies 1a and 1b separately can be found on OSF. Other research with this data has examined the ability to forecast need satisfaction in goal pursuit (Werner \& Milyavskaya, 2018), the distinct effects of personal standards and self-critical perfectionism on attributions of success and failure in goal pursuit (Levine, Werner, Capaldi, \& Milyavskaya, 2017), as well as trait self-control as a predictor of implementation intentions (Werner, Sjåstad, Milyavskaya, \& Hofmann, under review). There is no overlap between the content and the hypotheses of the present study and the other studies that have used this sample.
} 
Goal Self-Concordance. Participants rated five items that related to their autonomous and controlled reasons for pursuing each goal. Controlled reasons included "because somebody else wants you to, or because you'll get something from someone if you do" (external) and "because you would feel ashamed if you didn't - you feel that you should try to accomplish this goal" (introjected). Autonomous reasons included "because you really believe it is an important goal to have" (identified), "because of the fun and enjoyment which the goal will provide you - the primary reason is simply your interest in the experience itself" (intrinsic), and "because it represents who you are and reflects what you value most in life" (integrated). Items were rated on a scale ranging from 1 (not at all for this reason) to 7 (completely for this reason). Consistent with previous research (e.g., Sheldon \& Elliott, 1999; Werner et al., 2016), a measure of goal self-concordance was calculated by subtracting the average of the controlled items from the average of the autonomous items for each goal, such that the score for self-concordance ranged from -6 to 6 . Consistent with previous research (Grégoire et al., 2012), an overall score of goal self-concordance was calculated by taking the average of the self-concordant scores across all three goals.

Trait Mindfulness. Participants completed the Mindful Attention and Awareness Scale (MAAS; Brown \& Ryan, 2003; $\alpha=.86$ ) as a measure of trait mindfulness. The MAAS has been used extensively in previous research (Brown et al., 2007). Participants responded to fifteen items using a scale ranging from 1 (almost always) to 7 (almost never). Sample items include: "It seems I am "running on automatic" without much awareness of what I'm doing" and "I find myself doing things without paying attention." Scores from each item were averaged to form the scale total; higher scores indicated higher trait mindfulness.

Table 1.

Descriptive Statistics and Correlations

\begin{tabular}{|c|c|c|c|c|c|c|c|c|}
\hline Variable & Scale & $M$ & $S D$ & Mindfulness & $\begin{array}{c}\text { T1 } \\
\text { GSC }\end{array}$ & $\begin{array}{c}\text { Goal } \\
\text { Progress }\end{array}$ & $\begin{array}{c}\text { T2 } \\
\text { GSC }\end{array}$ & $\begin{array}{c}\text { GSC } \\
\text { Change }\end{array}$ \\
\hline \multicolumn{9}{|l|}{ Study 1} \\
\hline Mindfulness & $1-7$ & 3.62 & 0.87 & - & - & - & - & - \\
\hline Goal Self-Concordance & $-6-6$ & 1.08 & 1.78 & $.11 * *$ & - & - & - & - \\
\hline \multicolumn{9}{|l|}{ Study 2} \\
\hline Mindfulness & $1-6$ & 3.88 & 0.81 & - & - & - & - & - \\
\hline T1 Goal Self-Concordance & $-6-6$ & 1.56 & 1.65 & $.19 * *$ & - & - & - & - \\
\hline Goal Progress & $1-7$ & 4.27 & 1.11 & .09 & $.15^{*}$ & - & - & - \\
\hline T2 Goal Self-Concordance & $-6-6$ & 1.19 & 1.56 & $.26 * * *$ & $.74 * * *$ & $.28 * * *$ & - & - \\
\hline $\begin{array}{l}\text { Goal Self-Concordance } \\
\text { Change Score }\end{array}$ & $-12-12$ & -0.32 & 1.17 & .05 & $-.43 * * *$ & $.16^{*}$ & $.29 * * *$ & - \\
\hline
\end{tabular}

Study 3

Mindfulness

T1 Goal Self-Concordance

Goal Progress

T2 Goal Self-Concordance

Goal Self-Concordance Change Score

Note. Mindfulness: higher scores reflect greater trait mindfulness; GSC = Goal self-concordance; higher scores reflect greater goal self-concordance; Goal progress: higher scores reflect greater goal progress; Goal self-concordance change score: higher scores reflect becoming more self-concordant.

$* p<.05, * * \mathrm{p}<.01, * * * \mathrm{p}<.001$ 


\section{Results and Brief Discussion}

Descriptive statistics and correlations for Study 1 are presented in Table 1. A simple linear regression analysis was conducted to test our hypothesis. As predicted, trait mindfulness was positively associated with self-concordant goal setting, $\beta=.11, b=.23,95 \%$ CI [.087; .372], $t(773)=3.16, p=.002, R_{2}=.013$. This finding provided initial (albeit weak) support for our hypothesis that mindful individuals set goals that are more self-concordant. In an attempt to provide further support for our first hypothesis, Study 2 replicated and extended these findings.

\section{Study 2}

Study 2 investigated the relation between mindfulness, goal self-concordance, and goal progress using a longitudinal design. Given that mindfulness is theorized to predict self-concordant goal setting, it is also possible that mindfulness indirectly promotes goal progress since self-concordant goals are more likely to be attained (Koestner et al., 2002; Koestner et al., 2008; Milyavskaya et al., 2015; Sheldon \& Elliot, 1999; Werner et al., 2016). Moreover, mindfulness is associated with many benefits that may facilitate goal pursuit and is often used as a tool for boosting individual and organizational productivity in the business sector (Hafenbrack, 2017; Hyland, Lee, \& Mills, 2015). Despite this trend, the relationship between mindfulness and personal goal progress has yet to be investigated empirically. Study 2 aimed to address this gap in the literature and test our first two hypotheses: that greater dispositional mindfulness would positively predict self-concordant goal setting (H1), and that mindfulness would indirectly predict goal progress through self-concordant goal setting (H2). Additionally, we examined whether mindfulness affects internalization by testing its effects on changes in self-concordance over time.

\footnotetext{
5 Other research with this data has examined the role of domain need satisfaction in autonomous goals (Milyavskaya, Nadolny, \& Koestner, 2014), goal support (Koestner, Powers, Milyavskaya, Carbonneau, \& Hope, 2015), trait perfectionism and changes in affect (Milyavskaya et al., 2014), changes in identity and intimacy development as a function of changes in values (Hope, Milyavskaya, Holding, \& Koestner, 2014), the aspirational content of goals on goal progress and vitality (Hope et al., 2014), and the effects of perfectionism on attributions of success and failure. There is no overlap between the content and the hypotheses of the present study and the other studies that have used this sample.

6 Although here we report the aggregated results for the mediation model, because participants provided multiple goals (and therefore goals are nested within-person), we also ran a 2-1-1 mediation
}

\section{Method}

\section{Participants and Procedure}

Data from a previously collected study was used to test these hypotheses. Participants in Study 2 were 240 university students who took part in a larger study on goal pursuit.5 Twenty-two participants were removed from analyses for not completing items at the followup time points (final $n=218 ; 77.5 \%$ women; $M$ age $=$ 20.43 years, $S D=3.42$ ). Trait mindfulness was measured at one time point only at the start of the Fall semester; participants completed the MAAS (Brown \& Ryan, 2003; $\alpha=.87)$. At the beginning of the next semester (in early January), participants set three personal goals that they were going to pursue over the semester and rated their motivation for each goal using the same items from Study 1, which were used to calculate an average score for goal self-concordance. At the end of the semester (in April), participants completed a follow-up survey asking them to indicate how much progress they made on each of their goals on a scale ranging from 1 (strongly disagree) to 7 (strongly agree). Items included "I have made a lot of progress towards this goal," "I feel like I am on track with my goal plan," and "I feel close to achieving this goal" (Koestner et al., 2002; Werner et al., 2016). An aggregate score of goal progress was calculated by taking the average score for goal progress across all three goals. Goal self-concordance was measured again at this final time point as well.

\section{Results and Brief Discussion}

Descriptive statistics and correlations are presented in Table 1. Results suggest that trait mindfulness was positively related to self-concordant goal setting, $\beta=$ $.19, b=.3795 \%$ CI $[.11 ; .63], t(217)=2.82, p=.005$, $R_{2}=.035$. To test our second hypothesis, we conducted a simple mediation analysis using PROCESS (Hayes, 2013).6 As presented in Figure 1, trait mindfulness was

model that found a non-significant indirect effect on the between (no within-person meditation can be estimated in such a model; see OSF for full results). However, upon considering the structure of the data further, specifically that trait mindfulness is a betweenperson variable and should therefore affect goals in general (rather than within-person variances in goal pursuit), we report the aggregated findings above and use this same analytic strategy in the subsequent study. In other research (Werner et al., under review), two of the authors use multilevel mediation to examine indirect paths between a trait (trait self-control) and goal attainment (via implementation intentions and obstacles); however, in that study 
positively associated with goal self-concordance ( $a=$ $.43, p<.01,95 \%$ CI $[.156, .703])$, which in turn marginally predicted goal progress $(b=.09, p=.06$, 95\% CI $[-.004, .188])$. The biased-corrected bootstrap confidence interval (based on 10,000 samples) for the indirect effect was marginally significant (albeit very small; $a b=.04,95 \%$ CI: $[-.002, .096])$. Neither the total $(c=.11, p=.230,95 \%$ CI $[-.073, .301])$ nor the direct effect $\left(c^{\prime}=.07, p=.440,95 \%\right.$ CI $\left.[-.116, .265]\right)$ of trait mindfulness on goal progress was significant.

Given that the dataset used for Study 2 included a measure of goal self-concordance at the end of the semester (when goal progress was measured), additional exploratory analyses were conducted to investigate whether trait mindfulness predicted increases in goal self-concordance over time. First a difference score was calculated by subtracting "Time 2" goal self-concordance (measured at the end of the semester) from "Time 1" goal-self-concordance (measured at the beginning of the semester). Although difference scores have gained a negative reputation in the field, recent research suggests that this reputation is unwarranted (Gollwitzer, Christ, \& Lemmer, 2014) and that difference scores are a viable, and sometimes preferential, option for examining changes over time (Castro \& Grimm, 2018). The goal self-concordance difference score was hierarchically regressed on Time 1 goal self-concordance (Step 1), and trait mindfulness (Step 2). Table 2 presents the full results of this analysis. After controlling for Time 1 goal self-concordance, mindfulness positively predicted changes in goal self-concordance over time $(\beta=.15, p$
$=.026$ ), explaining $2.1 \%$ of the variance. We also investigated changes in goal self-concordance over time using the residualized change approach and the standardized residual approach and found similar results (see output on OSF).

Study 2 provided additional support for our first hypothesis that mindful individuals tend to set goals that are more self-concordant. However, results for our second hypothesis that mindfulness would indirectly predict goal progress through goal self-concordance were inconclusive. Given that the $b$ path in our mediation model was only marginally significant, and the confidence interval for the indirect effect just barely included zero, we sought to test the mediation hypothesis again in another data set.

Exploratory analyses also revealed that trait mindfulness predicted becoming more self-concordant over time. That is, participants rated their personal goals as increasingly more self-concordant over the course of the semester. Although this finding was exploratory, it reflects the processes of internalization described in self-determination theory (i.e., the process of internalizing goals, values, or behaviours often derived from external sources so that they are experienced as more volitional; Ryan \& Deci, 2017). Self-determination theory posits that mindful awareness facilitates this process (Ryan \& Deci, 2017), but this has yet to be empirically tested. While Study 2 provided initial empirical support for this proposition, we sought to replicate this finding in another dataset as well.
Study 2

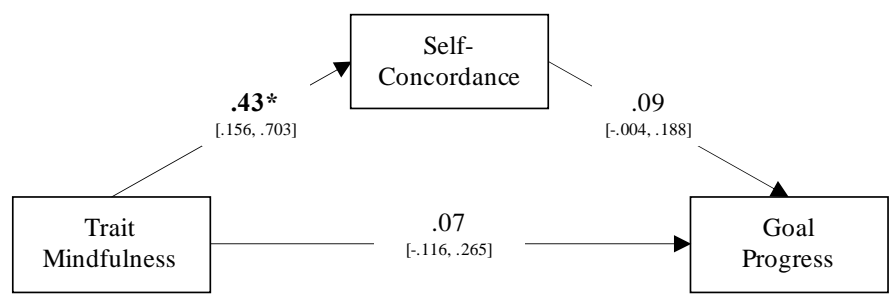

Indirect effect: $\mathrm{ab}=.04,95 \% \mathrm{CI}:[-.002, .096]$

Total effect: $c=.11,95 \%$ CI: $[-.073, .301]$
Study 3

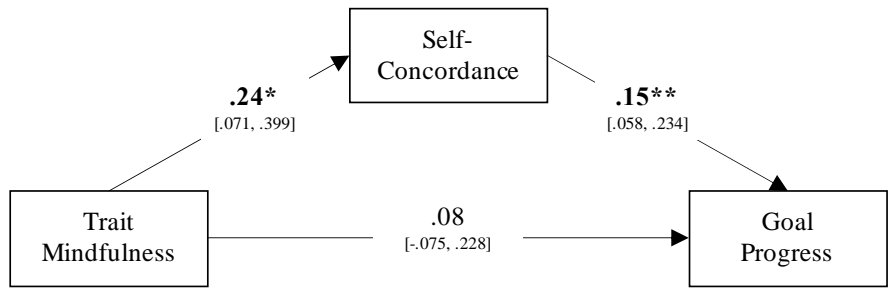

Indirect effect: $\mathrm{ab}=.03,95 \% \mathrm{CI}:[.006, .074]$

Total effect: $\mathrm{c}=.11,95 \%$ CI: $[-.041, .263]$

Figures 1 and 2. Mediation model depicting the relation between trait mindfulness and goal progress as mediated by goal selfconcordance. Unstandardized coefficients are presented (see manuscript for standardized). ${ }^{*} p<.05, * * p<.01$

there were two within-person mediators, such that a within-person indirect effect could be computed (in addition to the betweenperson indirect effect). Since this was not the case for the present study (i.e., only a between-person mediational pathway could have been computed), here we decided to ultimately proceed with aggregating the goal variables. 
Table 2.

Hierarchical Regression Analyses of Mindfulness on Changes in Goal Self-Concordance

\begin{tabular}{|c|c|c|c|c|c|c|c|c|}
\hline & $F$ & $R_{2}$ & $\Delta R_{2}$ & $b$ & 95\% C.I. & $\beta$ & $t$ & $p$ \\
\hline \multicolumn{9}{|l|}{ Study 2} \\
\hline Step 1 & $F(1,196)=44.94$ & .19 & .19 & & & & & $<.001$ \\
\hline T1 GSC & & & & -.30 & {$[-.39 ;-.22]$} & -.43 & -6.70 & $<.001$ \\
\hline Step 2 & $F(2,195)=25.46$ & .21 & .02 & & & & & $<.001$ \\
\hline T1 GSC & & & & -.33 & {$[-.42 ;-.24]$} & -.46 & -7.10 & $<.001$ \\
\hline Mindfulness & & & & .21 & {$[.03 ; .39]$} & .15 & 2.25 & .026 \\
\hline \multicolumn{9}{|l|}{ Study 3} \\
\hline Step 1 & $F(1,416)=36.03$ & .08 & .08 & & & & & $<.001$ \\
\hline T1 GSC & & & & -.32 & {$[-.43 ;-.22]$} & -.28 & -6.00 & $<.001$ \\
\hline Step 2 & $F(2,415)=28.31$ & .12 & .04 & & & & & $<.001$ \\
\hline T1 GSC & & & & -.35 & {$[-.46 ;-.25]$} & -.31 & -6.67 & $<.001$ \\
\hline Mindfulness & & & & .40 & {$[.22 ; .57]$} & .20 & 4.36 & $<.001$ \\
\hline
\end{tabular}

Note: GSC $=$ Goal self-concordance

\section{Study 3}

Study 3 served as a replication of Study 2 . It again tested the relationship between mindfulness and self-concordant goal setting, as well as the indirect effect of mindfulness on goal progress, via goal self-concordance.7 Study 3 also investigated whether mindfulness predicted increases in goal selfconcordance over time.

\section{Method}

\section{Participants and Procedure}

Data from a previously collected study was used to test our hypotheses. Participants in Study 3 were 508 university students $(82 \%$ women; $M$ age $=21.16$ years, $S D=3.99$ ) who took part in a larger study on goal pursuit.8 At the start of the fall semester, participants set three personal goals, rated their motivation for each goal (using the same items from our previous studies). Trait mindfulness was also measured at this time point only; participants completed an 8-item version of the MAAS (Brown \& Ryan, 2003; $\alpha=.77$ ). At the end of the academic year, participants reported on their progress for each goal (using the same items as Study 2) as well as their goal self-concordance once again.

\footnotetext{
7 We also investigated subjective well-being as an outcome variable (see supplemental materials) but did not include it here text since it was also investigated in Grégoire et al. (2012), albeit using a cross-sectional design.

8 Other research with this data has examined young adults' sacrifice of basic psychological needs in pursuit of career goals (Holding, StJacques,Verner-Filion, Kachanoff, \& Koestner, 2019), the benefits of matching personality traits with goal type for goal progress (Moore,
}

\section{Results and Brief Discussion}

Descriptive statistics and correlations are presented in Table 1. As predicted, trait mindfulness was positively associated with self-concordant goal setting, $\beta=.16, b=.29,95 \%$ CI $[.13 ; .44], t(507)=3.65, p<$ $.001, R_{2}=.026$. To test our second hypothesis, we conducted a simple mediation analyses using PROCESS (Hayes, 2013). As presented in Figure 2, trait mindfulness was positively associated with goal self-concordance $(a=.24, p<.01,95 \%$ CI [.071, $.399])$, which in turn predicted goal progress $(b=.15$, $p<.005,95 \%$ CI $[.058, .234])$. The biased-corrected bootstrap confidence interval (based on 10,000 samples) for the indirect effect was significant (albeit very small; $a b=.03,95 \%$ CI: $[.006, .074])$. Neither the total $(c=.11, p=.152,95 \%$ CI $[-.041, .263])$ nor the direct effect $\left(c^{\prime}=.08, p=.320,95 \%\right.$ CI $\left.[-.075, .228]\right)$ of trait mindfulness on goal progress was significant.

Once again, a hierarchical regression analysis was conducted to investigate whether mindfulness would predict increases in goal self-concordance over time. A difference score was calculated by subtracting Time 2 goal self-concordance from Time 1 goal self-concordance. Table 2 presents the full results of this analysis. After controlling for Time 1 goal

Holding, Verner-Filion, Harvey, \& Koestner, 2019), as well as the relationship between intrinsic goals and well-being via psychological need satisfaction and autonomous motivation (Hope, Holding, Verner-Filion, Sheldon, \& Koestner, 2019). There is no overlap between the content and the hypotheses of the present study and the other studies that have used this sample. 
self-concordance, mindfulness positively predicted changes in goal self-concordance over time $(\beta=.20, p$ $<.001$ ), accounting for $4 \%$ of the variance. We also investigated changes in goal self-concordance over time using the residualized change approach and the standardized residual approach and found similar results (see output on OSF).

Study 3 served as a replication of Study 2 and provided additional support for our hypotheses that mindful individuals would set goals that were more self-concordant (H1), and that this, in turn, would lead to greater goal progress (H2).9 Moreover, Study 3 provided additional support for the proposition that mindfulness promotes increases in goal self-concordance (i.e., internalization) over time.

\section{Meta-Analysis}

To corroborate our findings supporting the hypothesis that mindfulness would be positively correlated with self-concordant goal setting, we conducted a mini meta-analysis (Goh, Hall, \& Rosenthal, 2016) of our three studies using fixed effects in which the mean effect size (i.e., mean correlation) was weighted by sample size. All correlations were Fisher's $z$ transformed for analyses and converted back to Pearson correlations for presentation. Overall, the effect was small but significant, $M r=.14, Z=5.45, p$ $<.001,95 \%$ CI: [.090, .188], two-tailed, such that individuals who are more mindful set goals that are more self-concordant.

\section{Discussion}

The purpose of the present research was to investigate the relation between trait mindfulness, self-concordant goal setting, and goal progress. Three studies tested whether trait mindfulness was positively associated with self-concordant goal setting. Consistent with our first hypothesis, all three studies and an internal mini meta-analysis of the data found that individuals with higher levels of trait mindfulness were more likely to set goals that were more self-concordant. Results for our second hypothesis were less consistent - although Study 3 found a significant indirect effect of mindfulness on goal progress through self-concordance, the results from study 2 were

9 Since goal progress and subjective well-being were moderately positively correlated, we also conducted exploratory analyses controlling for the effect of subjective well-being (see supplemental materials on OSF). Mindfulness no longer had an indirect effect on goal progress when subjective well-being was included in the analysis as a covariate. Thus, the marginal; in both studies the indirect effect was small.

The positive relationship between mindfulness and self-concordant goal setting may be explained in part by self-awareness (Kudesia, 2019). Mindfulness promotes accurate self-awareness, which is essential for knowing which goals are self-appropriate (Brown \& Ryan, 2003; Sheldon, 2014). According to self-determination theory, awareness has long been posited as a foundational element to eudaimonic living because it facilitates the selection of goals and behaviours that are consistent with people's values, interests, and needs (Deci \& Ryan, 1980). By habitually paying attention to their thoughts, feelings, sensations, and emotions, mindful individuals may develop a greater ability to recognize goals that are congruent with their authentic selves. Additional support for this notion comes from research that reports that mindfulness can promote greater congruence between implicit and explicit self-esteem (Koole, Govorun, Cheng, \& Gallucci, 2009), suggesting that mindfulness may help to bring self-related expressions (e.g., self-esteem, personal goals) into greater alignment with their authentic selves.

Mindfulness may also promote goal selfconcordance through emotional processes. Although negative affect is associated with various cognitive, motivational, and interpersonal benefits (Forgas, 2013), it can also be problematic when it comes to effective decision-making (Loewenstein \& Lerner, 2002). Mindfulness is associated with reduced negative affect (Brown \& Ryan, 2003; Gu et al., 2015), and in turn, improved decision-making. For example, in a series of correlational and experimental studies, Hafenbrack, Kinias, and Barsade (2014) found that both trait and state mindfulness improved decision-making and that this effect was partially mediated by a reduction in negative affect. Given that mindful individuals experience relatively less negative affect, they may be more likely to make important decisions regarding which goals to pursue, in a tranquil state.

While the present research demonstrated a positive relationship between trait mindfulness and selfconcordant goal setting, it is important to note that the effect sizes were consistently small, particularly in comparison to previous research. Grégoire et al. (2012) reported a correlation of $r=.44$ between trait

indirect effect of mindfulness on goal progress may be explained in part by the overlap between goal progress and subjective well-being. 
mindfulness and self-determined goals whereas the meta-analysis in the present research reports a correlation of $r=.14$ between mindfulness and self-concordance. The discrepancy between these findings may be explained in part by the differences in the nature of the goals considered in each study. The goals reported in Grégoire et al. (2012) appear to have been long-term goals; examples include: "complete my bachelor's degree" and "learn to play guitar." These goals would arguably take years to pursue and achieve.

In the present research, goals ranged from weeklong (Study 1) to (academic) yearlong (Study 3). Taken together, mindfulness may correlate with self-concordance to a greater degree for long-term goals that span multiple years and are more reflective of individuals' core sense of self. Future research can examine whether the relationship between mindfulness and goal self-concordance varies as a function of the type of goal (e.g., short- vs. long-term, concrete vs. abstract).

In addition, Grégoire et al. (2012) had participants report their motivation for goals they were already pursuing, whereas the present research focused on newly set goals. In Studies 2 and 3, exploratory analyses revealed that mindful individuals appear to become more self-concordant in the pursuit of their goals over time. Thus, in Grégoire et al. (2012) individuals with higher trait mindfulness may have reported greater self-determination because they had already been pursuing their goals for some time, during which their motivation gradually shifted to become more internalized and self-concordant. Finally, although different formulas were used to calc ulate "self-determination" in Grégoire et al. (2012) and self-concordance in the present research, this did not appear to be an important factor in the differences in effect sizes. 10

Another potential explanation for the small effect sizes is that mindfulness merely promotes awareness of self-appropriate goals, which, on its own may not be a strong predictor of the selection of self-concordant goals. For example, in another study that used the same dataset as Study 2, authentic awareness (an aspect of authenticity) was not related to goal self-concordance (Milyavskaya, Nadolny, \& Koestner, 2015). According to Sheldon (2014) self-concordance is enhanced by factors that promote accurate self-insight (e.g., mindfulness) and factors that promote personal empowerment. Thus, it is possible that mindful

10 We conducted exploratory analyses using the same formula used in Grégoire et al., (2102) so that we could more easily make comparisons across studies (see "Formula Comparison" document individuals are more aware of self-concordant goal options but do not always possess the courage to select them.

The small effect sizes found in the present research may also relate to the level of analysis. Recent research suggests that the majority of the variance in goal characteristics exists at the goal level (i.e., within-person; Milyavskaya \& Werner, 2018). For example, previous research has shown that $60 \%$ to $80 \%$ of the variance in goal motivation exists at the within-person level (Holding et al., 2017; Milyavskaya et al., 2015; Werner et al., 2016). The present research investigated the relationship between mindfulness and self-concordance at the between-person level, since mindfulness is an individual difference, and is not goal specific. This approach was also used in Grégoire et al. (2012). From a theoretical standpoint, trait mindfulness is expected to facilitate the selection of goals and behaviours that are congruent with one's needs, values, and interests (Brown \& Ryan, 2003). Therefore, one would expect individuals who report higher trait mindfulness, to report greater self-concordance, on average across their various pursuits (i.e., collapsed across goals), than individuals who report lower levels of mindfulness. Practically, however, the high withinperson variability in both motivation and progress means that there may have been little between-person variance for mindfulness to explain.

Another potential explanation for the small effect sizes in the present research may be connected to the mindfulness measure that was used. In all three studies, the MAAS (Brown \& Ryan, 2003) was used. This measure was developed within the context of self-determination theory, from which the self-concordant model was derived (Sheldon \& Elliot, 1999). Although the MAAS (Brown and Ryan, 2003) is used extensively, it is comprised of fifteen reverse-coded items that emphasize acting without attention and awareness. Thus, it is possible that these items do not accurately reflect acting with attention and awareness and instead represent another construct (e.g., a judgmental and critical stance towards oneself; Bergomi, Tschacher, \& Zupper, 2013). Other mindfulness scales, such as the Five Facet Mindfulness Questionnaire (Baer, Smith, Hopkins, Krietemeyer, \& Toney, 2006) emphasize additional facets of mindfulness, including nonreactivity to inner experience, observing (thoughts, sensations, perceptions, feelings), describing with words, and

in the supplemental materials). The "self-determination" formula used in Grégoire et al., (2102) generated very similar results. 
nonjudging of experience. While there are several instruments for measuring trait mindfulness, considerable heterogeneity exists between these scales, and correlations between them typically range from .21 to 67 (Baer et al., 2006; Cardaciotto, Herbert, Forman, Moitra, \& Farrow, 2008). Therefore, it is possible that other measures or facets of mindfulness, particularly those relevant to observing one's internal world, would have stronger relationships with self-concordant goal selection.

Although Sheldon (2014) and self-determination theory propose that mindful awareness should facilitate the selection of self-concordant goals (and a recent meta-analysis that included mindfulness interventions provides support for this notion of directionality; Donald et al., 2019), it is worth considering the potential bidirectional nature of this relationship. For example, it is possible that individuals who set goals that are less self-concordant may shy away from attending to their internal world for fear that this discrepancy between their goals and their values and interests may lead them to feel sad, angry, guilty, or ashamed. While deploying mindful attention may feel rewarding when pursuing self-concordant goals, since these goals are considered to be important and interesting, it may be uncomfortable to deploy such attention in the pursuit of non-concordant goals as they can feel incongruent and coerced. Somewhat paradoxically, mindfulness may be particularly helpful in such a situation because mindfulness can enhance distress tolerance - the capacity for tolerating uncomfortable emotions or internal states (Robins et al., 2012). By openly and nonjudgmentally observing their internal states, without criticism or reprimand, these individuals may come to recognize this discrepancy as the source of their internal conflict, which in turn may prompt them towards greater self-concordance in the future.

Exploratory analyses in Studies 2 and 3 suggest that mindful individuals became more volitional, or self-concordant, over the course of their goal pursuit (i.e., their reasons for pursuing their goals became more autonomous and less controlled). In other words, mindful individuals were more likely to internalize their goals. According to self-determination theory, internalization involves "a process through which, often by being mindful and using higher-order reflection, people are able to bring externally imposed, value-based action into the realm of fully volitional activity" (Ryan \& Deci, 2017, p. 189). Studies 2 and 3 provide support for the proposition that mindful awareness facilitates internalization. By deploying mindful attention and adopting an open and receptive attitude over the course of their goal pursuit, mindful individuals may have experienced greater interest in, and enjoyment of, their goals (perhaps through enhanced present-moment experiences; Killingsworth \& Gilbert, 2010). This explanation would be consistent with previous research suggesting that mindful individuals report greater interest in day-to-day activities such as social interactions with romantic partners (Barnes, Brown, Krusemark, Campbell, \& Rogge, 2007) and everyday work tasks (Shiba et al., 2015). It is also possible that mindful individuals were better able to recognize the value of their goals while pursuing them. Indeed, previous research suggests that trait mindfulness is positively associated with identified motivation (i.e., pursuing goals because they are meaningful; Donald et al., 2019). By taking greater interest in, and/or further recognizing the importance of, their goals, mindful individuals may come to more fully endorse them over the course of their goal pursuit. On the other hand, individuals with lower levels of trait mindfulness may fail to take notice of the positive qualities of their goal pursuit, and instead, may be more apt to regard their goals as things they have to do.

Although a large body of research supports the notion that mindfulness is associated with more adaptive motivational qualities (Donald et al., 2019), the present research offers two novel contributions to this literature. First, whereas previous research has mostly focused on mindfulness and its relation to participants' motivation towards goals assigned by researchers (Ryan \& Deci, 2017), the present research provides insight into the types of goals that mindful individuals are likely to set on their own, as well as their motivation towards these goals. Second, while a large portion of the research on mindfulness and motivation has focused on participants' motivation towards behaviours and goals that they were already engaged in, or that they typically engage in (e.g., Brown \& Ryan, 2003; Gregoire et al., 2012; Christie, Atkins, \& Donald et al., 2017; Warren \& Wray-Lake, 2017), the present research focused on new goals that participants had yet to pursue. Given that motivation can shift over time and that mindfulness, as the present research suggests, may promote greater goal internalization, the present findings may help to more appropriately interpret previous findings related to individuals' motivation towards behaviours and goals that they were already engaged with. That is, in previous research, where correlations may be relatively larger than the correlations observed in the present research, with respect to participants' initial motivation towards their 
newly set goals, the larger correlations may be due in part to some degree of goal internalization over time.

The present research also suggests that mindfulness may have a small indirect effect on goal progress via goal self-concordance. To the extent that mindful individuals set goals that are more self-concordant, they may be able to make greater progress on their goals. This is consistent with previous research that has found that pursuing self-concordant goals leads to greater goal progress through increased effort (Koestner et al., 2002; Koestner et al., 2008; Sheldon \& Elliot 1999), subjective ease (Werner et al., 2016), and fewer obstacles and temptations (Milyavskaya et al., 2015). Conversely, there was no evidence of a direct or total effect of mindfulness on goal progress in Studies 2 and 3. This finding is noteworthy given that mindfulness is often portrayed in the media as an effective tool for boosting productivity (Pinsker, 2015). Additionally, this finding may lend credence to previous research that suggests mindfulness may not enhance motivation and performance in all contexts (Hafenbrack \& Vohs, 2018). Nonetheless, mindfulness is associated with a host of beneficial outcomes that may facilitate goal pursuit (Friese \& Hofmann, 2016; Hafenbrack \& Vohs, 2018; Weinstein et al., 2009); therefore, this finding must be interpreted with caution since it was not hypothesized and was only considered post-hoc. In addition, personal goal progress was measured using self-report, which is subjective in nature. It is possible that mindful individuals are more willing to admit failures and disclose poor goal progress, which could be uncomfortable or threatening to one's self-esteem. Support for this idea may come from research that reports that mindfulness is associated with more adaptive (e.g., less defensive) responses to existential threats (Niemiec et al., 2010), social-evaluative threats (Brown, Weinstein, \& Creswell, 2012), and ego threats (Bergeron \& Dandeau, 2016). Given that this was the first study to investigate mindfulness and goal progress, more research (with objective measures of goal progress) is needed to elucidate this relationship.

\section{Limitations and Future Directions}

It is important to note that the present research was non-experimental and therefore, no causal conclusions can be made from the results. Despite the strong theoretical rationale for the notion that mindfulness

11 Supplementary analyses showed that trait mindfulness no longer predicted Time 1 goal self-concordance after controlling for Time 1 subjective well-being but that that trait mindfulness still predicted Time 2 may promote greater goal self-concordance, it is possible that the relationship between trait mindfulness and goal self-concordance could be explained in part by third variables related to affect and well-being, rather than an underlying causal relationship. For example, both trait mindfulness (Brown \& Ryan, 2003) and self-concordance (Sheldon \& Elliot, 1999) have been positively associated with subjective well-being. As we found in supplementary analyses,11 the relationship between trait mindfulness and goal self-concordance may be due at least in part to the common correlation between each of these variables and subjective well-being.

Another limitation is that the effects sizes for the relationships between trait mindfulness, goal self-concordance, and goal progress were consistently very small. Moreover, the MAAS (Brown \& Ryan, 2003) was the only measure used to investigate trait mindfulness. Other mindfulness measures or facets of mindfulness may have stronger relationships with goal self-concordance and goal progress (Park, Reilly-Spong, \& Gross, 2013). Similarly, although self-concordance was assessed on a continuum following the most frequently used method (Sheldon \& Elliot, 1999), others have examined autonomous and controlled motivation separately (e.g., Milyavskaya et al., 2015) or more recently recommended assessing self-concordance in a slightly different way (Sheldon, Osin, Gordeeva, Suchkov, \& Sychev, 2017); such distinctions may lead to small differences in the results. Furthermore, the present research consisted of undergraduate samples; therefore, findings may not necessarily generalize to younger or older populations.

Future research should investigate whether interventions designed to enhance trait mindfulness can improve individuals' ability to set self-concordant goals. A recent meta-analysis that included mindfulness interventions found a medium-sized effect from mindfulness interventions to autonomous motivation (Donald et al., 2019). To the extent that we are able to increase an individual's dispositional mindfulness, we may be able to improve his or her ability to set self-concordant goals. This is important given that people are often "out of touch" with what they truly want (Sheldon \& Kasser, 1995), and previous interventions designed to increase self-concordance have failed to produce the desired effect (Sheldon, Kasser, Smith \& Share, 2002). It is important to note, however, that the effects in the present research were

goal self-concordance after controlling for Time 2 subjective well-being (see supplemental materials on OSF). 
very small, so any potential changes from interventions may be modest.

Lastly, more research is needed to shed light on the relationship between mindfulness and goal progress. The recent trend to use mindfulness as a tool to boost productivity typically involves the use of mindfulness exercises such as mindfulness meditation. Future research could employ experimental designs to determine whether mindfulness interventions can enhance progress on individuals' personal goals.

\section{Conclusion}

The present research demonstrated and replicated a small positive relationship between trait mindfulness and self-concordant goal setting (Studies 1-3). We also found (partial) support for the notion that trait mindfulness promotes goal progress indirectly through goal self-concordance. Finally, in line with self-determination theory, Studies 2 and 3 found that trait mindfulness predicted increases in goal internalization over the course of individuals' goal pursuit. Taken together, this research provides support for the proposition that mindful individuals set "better" goals - goals that are more likely to be aligned with their authentic selves, conducive to goal progress, and internalized over time.

\section{References}

Allexandre, D., Bernstein, A. M., Walker, E., Hunter, J., Roizen, M. F., \& Morledge, T. J. (2016). A web-based mindfulness stress management program in a corporate call center: a randomized clinical trial to evaluate the added benefit of onsite group support. Journal of occupational and environmental medicine, 58(3), 254-264.

Baer, R. A., Smith, G. T., Hopkins, J., Krietemeyer, J., \& Toney, L. (2006). Using self-report assessment methods to explore facets of mindfulness. Assessment, 13(1), 27-45.

Barnes, S., Brown, K. W., Krusemark, E., Campbell, W. K., \& Rogge, R. D. (2007). The role of mindfulness in romantic relationship satisfaction and responses to relationship stress. Journal of marital and family therapy, 33(4), 482-500.

Bergeron, C. M., \& Dandeneau, S. (2016). Implicitly activating mindfulness promotes positive responses following an ego threat. Journal of Social and Clinical Psychology, 35(7), 551570.

Bergomi, C., Tschacher, W., \& Kupper, Z. (2013). The assessment of mindfulness with self-report measures: Existing scales and open issues. Mindfulness, 4(3), 191-202.

Brown, K. W., \& Ryan, R. M. (2003). The benefits of being present: mindfulness and its role in psychological well-being. Journal of personality and social psychology, 84(4), 822.

Brown, K. W., Ryan, R. M., \& Creswell, J. D. (2007). Mindfulness: Theoretical foundations and evidence for its salutary effects. Psychological inquiry, 18(4), 211-237.
Brown, K. W., Weinstein, N., \& Creswell, J. D. (2012). Trait mindfulness modulates neuroendocrine and affective responses to social evaluative threat. Psychoneuroendocrinology, 37(12), 2037-2041.

Burton, C. M. (2008). Gut feelings and goal pursuit: A path to selfconcordance. Dissertation Abstracts International: Section B. Sciences and Engineering, 73(2-B), 1303-1387.

Cardaciotto, L., Herbert, J. D., Forman, E. M., Moitra, E., \& Farrow, V. (2008). The assessment of present-moment awareness and acceptance: The Philadelphia Mindfulness Scale. Assessment, 15(2), 204-223.

Castro-Schilo, L., \& Grimm, K. J. (2018). Using residualized change versus difference scores for longitudinal research. Journal of Social and Personal Relationships, 35(1), 32-58.

Christie, A. M., Atkins, P. W., \& Donald, J. N. (2017). The meaning and doing of mindfulness: The role of values in the link between mindfulness and well-being. Mindfulness, 8(2), 368378.

Converse, B. A., Juarez, L., \& Hennecke, M. (2019). Self-control and the reasons behind our goals. Journal of personality and social psychology, 116(5), 860-883.

Deci, E. L., \& Ryan, R. M. (1980). Self-determination theory: When mind mediates behavior. The Journal of Mind and Behavior, 1(1), 33-43.

Deci, E. L., \& Ryan, R. M. (2000). The" what" and" why" of goal pursuits: Human needs and the self-determination of behavior. Psychological inquiry, 11(4), 227-268.

Diener, E. D., Emmons, R. A., Larsen, R. J., \& Griffin, S. (1985). The satisfaction with life scale. Journal of personality assessment, 49(1), 71-75.

Donald, J. N., Bradshaw, E. L., Ryan, R. M., Basarkod, G., Ciarrochi, J., Duineveld, J. J., ... \& Sahdra, B. K. (2019). Mindfulness and its association with varied types of motivation: A systematic review and meta-analysis using selfdetermination theory. Personality and Social Psychology Bulletin.

Forgas, J. P. (2013). Don't worry, be sad! On the cognitive, motivational, and interpersonal benefits of negative mood. Current Directions in Psychological Science, 22(3), 225-232.

Friese, M., \& Hofmann, W. (2016). State mindfulness, selfregulation, and emotional experience in everyday life. Motivation Science, 2(1), 1-14.

Goh, J. X., Hall, J. A., \& Rosenthal, R. (2016). Mini meta-analysis of your own studies: Some arguments on why and a primer on how. Social and Personality Psychology Compass, 10(10), 535 549.

Gollwitzer, M., Christ, O., \& Lemmer, G. (2014). Individual differences make a difference: On the use and the psychometric properties of difference scores in social psychology. European Journal of Social Psychology, 44(7), 673-682.

Gu, J., Strauss, C., Bond, R., \& Cavanagh, K. (2015). How do mindfulness-based cognitive therapy and mindfulness-based stress reduction improve mental health and wellbeing? A systematic review and meta-analysis of mediation studies. Clinical psychology review, 37, 1-12.

Hafenbrack, A. C. (2017). Mindfulness meditation as an on-thespot workplace intervention. Journal of Business Research, 75, 118-129.

Hafenbrack, A. C., Kinias, Z., \& Barsade, S. G. (2014). Debiasing the mind through meditation: Mindfulness and the sunk-cost bias. Psychological science, 25(2), 369-376. 
Hafenbrack, A. C., \& Vohs, K. D. (2018). Mindfulness meditation impairs task motivation but not performance. Organizational Behavior and Human Decision Processes, 147, 1-15.

Hayes, A. F. (2013). Introduction to mediation, moderation, and conditional process analysis: A regression-based approach. 2013. Guilford. New York.

Holding, A. C., Hope, N. H., Harvey, B., Marion Jetten, A. S., \& Koestner, R. (2017). Stuck in limbo: Motivational antecedents and consequences of experiencing action crises in personal goal pursuit. Journal of Personality, 85(6), 893-905

Holding, A., Hope, N., Verner-Filion, J., \& Koestner, R. (2019). In good time: A longitudinal investigation of trait self-control in determining changes in motivation quality. Personality and Individual Differences, 139, 132-137.

Holding, A. C., St-Jacques, A., Verner-Filion, J., Kachanoff, F., \& Koestner, R. (2019). Sacrifice-but at what price? A longitudinal study of young adults' sacrifice of basic psychological needs in pursuit of career goals. Motivation and Emotion, 1-17.

Hope, N. H., Holding, A. C., Verner-Filion, J., Sheldon, K. M., \& Koestner, R. (2019). The path from intrinsic aspirations to subjective well-being is mediated by changes in basic psychological need satisfaction and autonomous motivation: A large prospective test. Motivation and Emotion, 43(2), 232-241.

Hope, N. H., Milyavskaya, M., Holding, A. C., \& Koestner, R. (2014). Self-growth in the college years: Increased importance of intrinsic values predicts resolution of identity and intimacy stages. Social psychological and personality science, 5(6), 705712.

Hyland, P. K., Lee, R. A., \& Mills, M. J. (2015). Mindfulness at work: a new approach to improving individual and organizational performance. Industrial and Organizational Psychology, 8(4), 576-602.

Kabat-Zinn, J. (2003). Mindfulness-based Interventions in context: past, present, and future. Clinical Psychology: Science and Practice, 10(2), 144-156.

Kersemaekers, W., Rupprecht, S., Wittmann, M., Tamdjidi, C., Falke, P., Donders, R., ... \& Kohls, N. (2018). A workplace mindfulness intervention may be associated with improved psychological well-being and productivity. A preliminary field study in a company setting. Frontiers in psychology, 9, 1-11.

Killingsworth, M. A., \& Gilbert, D. T. (2010). A wandering mind is an unhappy mind. Science, 330(6006), 932-932.

Koestner, R., Lekes, N., Powers, T. A., \& Chicoine, E. (2002). Attaining personal goals: Self-concordance plus implementation intentions equals success. Journal of personality and social psychology, 83(1), 231-244.

Koestner, R., Otis, N., Powers, T. A., Pelletier, L., \& Gagnon, H. (2008). Autonomous motivation, controlled motivation, and goal progress. Journal of personality, 76(5), 1201-1230.

Koestner, R., Powers, T. A., Milyavskaya, M., Carbonneau, N., \& Hope, N. (2015). Goal internalization and persistence as a function of autonomous and directive forms of goal support. Journal of Personality, 83(2), 179-190.

Koole, S. L., Govorun, O., Cheng, C. M., \& Gallucci, M. (2009). Pulling yourself together: Meditation promotes congruence between implicit and explicit self-esteem. Journal of Experimental Social Psychology, 45(6), 1220-1226.

Kudesia, R. S. (2019). Mindfulness as metacognitive practice. Academy of Management Review, 44(2), 405-423.

Levine, S. L., Werner, K. M., Capaldi, J. S., \& Milyavskaya, M. (2017). Let's play the blame game: The distinct effects of personal standards and self-critical perfectionism on attributions of success and failure during goal pursuit. Journal of Research in Personality, 71, 57-66.

Loewenstein, G., \& Lerner, J. S. (2002). The role of affect in decision making. In R. Davidson, K. Scherer, \& H. Goldsmith (Eds.), Handbook of affective science (pp. 619-642). New York, NY: Oxford University Press.

Milyavskaya, M., Inzlicht, M., Hope, N., \& Koestner, R. (2015). Saying "no" to temptation: Want-to motivation improves selfregulation by reducing temptation rather than by increasing self-control. Journal of Personality and Social Psychology, 109(4), 677-693.

Milyavskaya, M., Nadolny, D., \& Koestner, R. (2015). Why do people set more self-concordant goals in need satisfying domains? Testing authenticity as a mediator. Personality and Individual Differences, 77, 131-136.

Milyavskaya, M., \& Werner, K. M. (2018). Goal pursuit: Current state of affairs and directions for future research. Canadian Psychology/Psychologie Canadienne, 59(2), 163-175.

Moore, A., Holding, A., Verner-Filion, J., Harvey, B., \& Koestner, R. (2020). A longitudinal investigation of trait-goal concordance on goal progress: The mediating role of autonomous goal motivation. Journal of Personality, 88(3), 350-543.

Moore, E., Holding, A. C., Hope, N. H., Harvey, B., Powers, T. A., Zuroff, D., \& Koestner, R. (2018). Perfectionism and the pursuit of personal goals: A self-determination theory analysis. Motivation and Emotion, 42(1), 37-49.

Niemiec, C. P., Brown, K. W., Kashdan, T. B., Cozzolino, P. J., Breen, W. E., Levesque-Bristol, C., \& Ryan, R. M. (2010). Being present in the face of existential threat: The role of trait mindfulness in reducing defensive responses to mortality salience. Journal of personality and social psychology, 99(2), 344-365.

Park, T., Reilly-Spong, M., \& Gross, C. R. (2013). Mindfulness: a systematic review of instruments to measure an emergent patient-reported outcome (PRO). Quality of Life Research, 22(10), 2639-2659.

Pinsker, J. (2015, March 10). Corporations' newest productivity hack: Meditation. The Atlantic Retrieved from https://www.theatlantic.com/business/archive/2015/03/corpora tions-newest-productivity-hack-meditation/387286/.

Robins, C., Keng, S., Ekblad, A., \& Brantley, J. (2012). Effects of mindfulness-based stress reduction on emotional experience and expression: A randomized controlled trial. Journal of Clinical Psychology, 68(1), 117-131.

Ryan, R. M., Kuhl, J., \& Deci, E. L. (1997). Nature and autonomy: An organizational view of social and neurobiological aspects of self-regulation in behavior and development. Development and psychopathology, 9(4), 701-728.

Shapiro, S. L., Carlson, L. E., Astin, J. A., \& Freedman, B. (2006). Mechanisms of mindfulness. Journal of clinical psychology, 62(3), 373-386.

Sheldon, K. M. (2014). Becoming oneself: The central role of selfconcordant goal selection. Personality and Social Psychology Review, 18(4), 349-365.

Sheldon, K. M., \& Elliot, A. J. (1999). Goal striving, need satisfaction, and longitudinal well-being: the self-concordance model. Journal of personality and social psychology, 76(3), 482-497.

Sheldon, K. M., \& Kasser, T. (1995). Coherence and congruence: Two aspects of personality integration. Journal of Personality and Social Psychology, 68(3), 531-543.

Sheldon, K. M., \& Kasser, T. (1998). Pursuing personal goals: Skills enable progress, but not all progress is 
beneficial. Personality and social psychology bulletin, 24(12), 1319-1331.

Sheldon, K. M., \& Kasser, T. (2001). Getting older, getting better? Personal strivings and psychological maturity across the life span. Developmental psychology, 37(4), 491-501.

Sheldon, K. M., Kasser, T., Smith, K., \& Share, T. (2002). Personal goals and psychological growth: Testing an intervention to enhance goal attainment and personality integration. Journal of Personality, 70(1), 5-31.

Sheldon, K. M., Osin, E. N., Gordeeva, T. O., Suchkov, D. D., \& Sychev, O. A. (2017). Evaluating the dimensionality of selfdetermination theory's relative autonomy continuum. Personality and Social Psychology Bulletin, 43(9), 1215-1238.

Sheldon, K. M., Prentice, M., Halusic, M., \& Schüler, J. (2015). Matches between assigned goal-types and both implicit and explicit motive dispositions predict goal selfconcordance. Motivation and Emotion, 39(3), 335-343.

Shiba, K., Nishimoto, M., Sugimoto, M., \& Ishikawa, Y. (2015). The association between meditation practice and job performance: A cross-sectional study. PloS one, 10(5). Warren, M. T., \& Wray-Lake, L. (2017). Does mindfulness prepare adolescents for value- behavior concordance? Examining the role of value content. Journal of Adolescence, 58, 56-66.

Watson, D., Clark, L. A., \& Tellegen, A. (1988). Development and validation of brief measures of positive and negative affect: the PANAS scales. Journal of personality and social psychology, 54(6), 1063-1070.
Weinstein, N., Brown, K. W., \& Ryan, R. M. (2009). A multimethod examination of the effects of mindfulness on stress attribution, coping, and emotional well-being. Journal of Research in Personality, 43(3), 374-385.

Werner, K. M. (2019). The Determinants of Successful Goal Pursuit (Doctoral dissertation, Carleton University).

Werner, K. M., \& Milyavskaya, M. (2018). We may not know what we want, but do we know what we need? Examining the ability to forecast need satisfaction in goal pursuit. Social Psychological and Personality Science, 9(6), 656-663.

Werner, K. M., Milyavskaya, M., Foxen-Craft, E., \& Koestner, R. (2016). Some goals just feel easier: Self-concordance leads to goal progress through subjective ease, not effort. Personality and Individual Differences, 96, 237-242.

Werner, K. M., Sjåstad, H., Milyavskaya, M., \& Hofmann, W. 2020). Planning for success: Trait self-control as a predictor of implementation intentions during goal pursuit. PsyArXiv. https://doi.org/10.31234/osf.io/m9u4e

Zhao, X., Lynch Jr, J. G., \& Chen, Q. (2010). Reconsidering Baron and Kenny: Myths and truths about mediation analysis. Journal of consumer research, 37(2), 197-206.

This pre-print was designed by KMW using the following template: Wiernik, B. M. (2019, October 11). Should you notice any differences between this file and the version of record, please cite the original published version.

Preprint templates. https://doi.org/10.17605/OSE.IO/HSV6A 